\title{
Second-harmonic generation in nonlinear left-handed metamaterials
}

\author{
Ilya V. Shadrivov ${ }^{1}$, Alexander A. Zharov ${ }^{1,2}$, and Yuri S. Kivshar ${ }^{1}$ \\ 1 Nonlinear Physics Center, Research School of Physical Sciences and Engineering, \\ Australian National University, Canberra ACT 0200, Australia \\ 2 Institute for Physics of Microstructures, Russian Academy of Sciences, Nizhny Novgorod 603950, Russia
}

\begin{abstract}
We study the second-harmonic generation in left-handed metamaterials with a quadratic nonlinear response. We demonstrate a novel type of the exact phase matching between the backward propagating wave of the fundamental frequency and the forward propagating wave of the second harmonics. We show that this novel parametric process can convert a surface of the left-handed metamaterial into an effective mirror totally reflecting the second harmonics generated by an incident wave. We derive and analyze theoretically the coupled-mode equations for a semi-infinite nonlinear metamaterial. We also study numerically the second-harmonic generation by a metamaterial slab of a finite thickness, and reveal the existence of multistable nonlinear effects.
\end{abstract}

\section{INTRODUCTION}

Recent years demonstrated many advances in the design and engineering of artificial composite structures with unique electromagnetic response, which have broadened significantly the range of possible wave phenomena not usually observed in Nature but being accessed in laboratory experiment. In particular, it has been shown that the composite metallic periodic structures may allow realizing hypothetical and surprising materials, first predicted theoretically and termed left-handed media [1], which possess simultaneously negative dielectric permittivity and magnetic permeability. Indeed, the composite metallic structures consisting of arrays of wires and splitring-resonators have been demonstrated to possess lefthanded properties in the microwave frequency range [2]. These composite materials received a great deal of interest due to their highly nontrivial and quite often counterintuitive electromagnetic properties. In particular, such materials support backward waves, and they exhibit negative refraction at interfaces [3].

The study of linear wave propagation and linear properties of left-handed materials is a major subject of research in this field. It is indeed the case when both magnetic permeability and dielectric permittivity of the material do not depend on the intensity of the electromagnetic field. However, the future efforts creating tunable structures where the field intensity changes the transmission properties of the composite structure would require the knowledge of nonlinear properties of such metamaterials, which may be quite unusual. Indeed, inserting small conducting elements into a periodic metallic structure of the metamaterial brings an unique opportunity to enhance nonlinear response in such left-handed media because the microscopic electromagnetic field inside the structure resonant elements can become much stronger than an average macroscopic field. Indeed, it has already been shown analytically and numerically that the composite material containing nonlinear elements in the slits of split-ring resonators possesses a hysteresis-type nonlinear magnetic response [4, 5]. This is an example of selfaction of electromagnetic waves usually associated with the cubic nonlinearity or its different generalizations.

Inclusion the elements with non-symmetric currentvoltage characteristics such as diodes into the split-ring resonators will result in a quadratic nonlinear response of the metamaterial [ $[$ ]. This quadratic nonlinearity is responsible for the recently analyzed parametric processes such as the second-harmonic generation (SHG) 7] and three-wave mixing [8]. In particular, the first analysis of SHG from a semi-infinite left-handed medium has been briefly presented by Agranovich et al. 7], who employed the nonlinear optics approach and also made a statement that for metamaterials "... SHG in transmission is badly phase-mismatched" parametric process, and "it is then the SHG in reflection that is more interesting". (see Ref. 7], p. 165112-5).

In this paper we consider the problem of SHG during the scattering from a semi-infinite left-handed medium (or a slab of the left-handed material of a finite extent) and demonstrate that the original paper by Agranovich et al. 7] missed an important additional phase-matching condition, quite specific for the harmonic generation by the backward waves. With this condition, we demonstrate that exact phase matching between a backward propagating wave of the fundamental frequency $(\mathrm{FF})$ and the forward propagating wave at the second harmonics $(\mathrm{SH})$ is indeed possible. This novel phase matched process allows for creating an effective "quadratic mirror" that reflects the $\mathrm{SH}$ component generated by an incident FF wave.

The paper is organized as follows. In Sec. 2 we describe our model including both the electric and magnetic responses. Quadratic nonlinearity and the SHG process in metamaterials are discussed in Sec. 3. In Sec. 4, we develop the corresponding coupled-mode theory for SHG with backward waves and present the analysis of both lossy and lossless cases of this model. Section 5 is devoted to the case of SHG process a slab of finite-extension. At last, Sec. 6 concludes the paper. 


\section{MODEL}

We consider a three-dimensional composite structure consisting of a cubic lattice of conducting wires and splitring resonators (SRR), shown schematically in the insert of Fig. 1 We assume that the unit-cell size of the structure $d$ is much smaller then the wavelength of the propagating electromagnetic field and, for simplicity, we choose a single-ring geometry of the lattice of SRRs. The results obtained for this case are qualitatively similar to those obtained in more involved cases of double SRRs. This type of microstructured medium is known to possess the basic properties of left-handed metamaterials exhibiting negative refraction in the microwave region.

In the effective-medium approximation, a response of this composite metallic structure can be described by averaged equations allowing to introduce the effective dielectric permittivity and effective magnetic permeability of the form

$$
\begin{gathered}
\epsilon(\omega)=1-\frac{\omega_{p}^{2}}{\omega^{2}}, \\
\mu(\omega)=1+\frac{F \omega}{\left(\omega_{0}^{2}-\omega^{2}\right)},
\end{gathered}
$$

where $\omega_{p}$ is the effective plasma frequency, $\omega_{0}$ is a resonant frequency of the array of SRRs, $F$ is the form-factor of the lattice, and $\omega$ is the angular frequency of the electromagnetic waves. The product of permittivity $\epsilon$ and permeability $\mu$ defines the square of the effective refractive index, $n^{2}=\epsilon \mu$, and its sign determines if waves can $\left(n^{2}>0\right)$ or cannot $\left(n^{2}<0\right)$ propagate in the medium. Due to the medium dispersion defined by the dependencies (11) and (2), the wave propagation becomes possible only in certain frequency domains while the waves decay for other frequencies. Metamaterial possesses lefthanded properties when both $\epsilon$ and $\mu$ become simultaneously negative, and such a frequency domain exists in the model described by Eqs. (1) and (2) provided $\omega_{p}>\omega_{0}$. In this case, the metamaterial is left-handed within the frequency range

$$
\omega_{0}<\omega<\min \left\{\omega_{p}, \omega_{M}\right\}, \omega_{M}=\frac{\omega_{0}}{\sqrt{1-F}},
$$

where $\omega_{p}$ is the plasma frequency introduced in Eq. (11).

We assume that $\omega_{M}<\omega_{p}$, and in this case we have two frequency ranges where the material is transparent, the range where the material is left-handed (LHM), and the right-handed (RHM) domain for $\omega>\omega_{p}$, where both permittivity and permeability are positive (shaded domains in Fig. 11). For the frequencies outside these two domains, the composite material is opaque.

\section{QUADRATIC NONLINEARITY AND BASIC EQUATIONS}

The composite material becomes nonlinear and it possesses a quadratic nonlinear response when, for example,

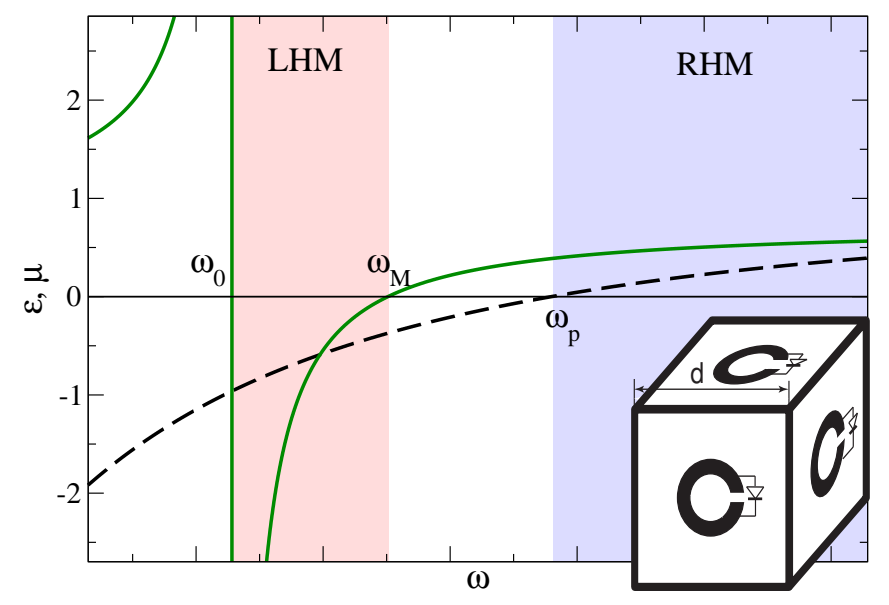

FIG. 1: Frequency-dependent magnetic permeability $\mu$ (solid) and electric permittivity $\epsilon$ (dashed) of the composite. Two types of the regions (LHM or RHM) where the material is transparent are shaded. For other frequencies it is opaque. Characteristic frequencies $\omega_{0}, \omega_{M}$, and $\omega_{p}$ are defined in Eqs. (11) to (3). Inset shows the unit cell of the metamaterial.

additional diodes are inserted into the SRRs of the structure [6], as shown schematically in the insert of Fig. 1] Quadratic nonlinearity is known to be responsible for various parametric processes in nonlinear media, including the frequency doubling and generation of the secondharmonic field. In dispersive materials, and especially in the metamaterials with the frequency domains with different wave properties, the SHG process can be rather nontrivial because the wave at the fundamental frequency and the second harmonics can fall into different domains of the material properties.

The most unusual harmonic generation and other parametric processes are expected when one of the waves (either FF or $\mathrm{SH}$ wave) has the frequency for which the metamaterial becomes left-handed. The specific interest to this kind of parametric processes is due to the fact that the waves in the left-handed media are backward, i.e. the energy propagates in the direction opposite to that of the wave vector. Both phase-matching condition and nonlinear interaction of the forward and backward waves may become quite nontrivial, as is known from the physics of surface waves in plasmas [9]. In this paper, we are interested in this type of parametric wave interactions.

In nonlinear quadratic composite metamaterials, interaction of the forward and backward waves of different harmonics takes place when the material is left-handed either for the frequency $\omega$ or the double frequency $2 \omega$. Under this condition, there exist two types of the most interesting SHG parametric processes in metamaterials.

Case $I$. The frequency of the FF wave is in the range $\omega_{0} / 2<\omega<\omega_{M} / 2$ and, therefore, the $\mathrm{SH}$ wave is generated with the double frequency in the LHM domain (see Fig. 1). For such parameters, the electromagnetic waves at the FF frequency are non-propagating, since 


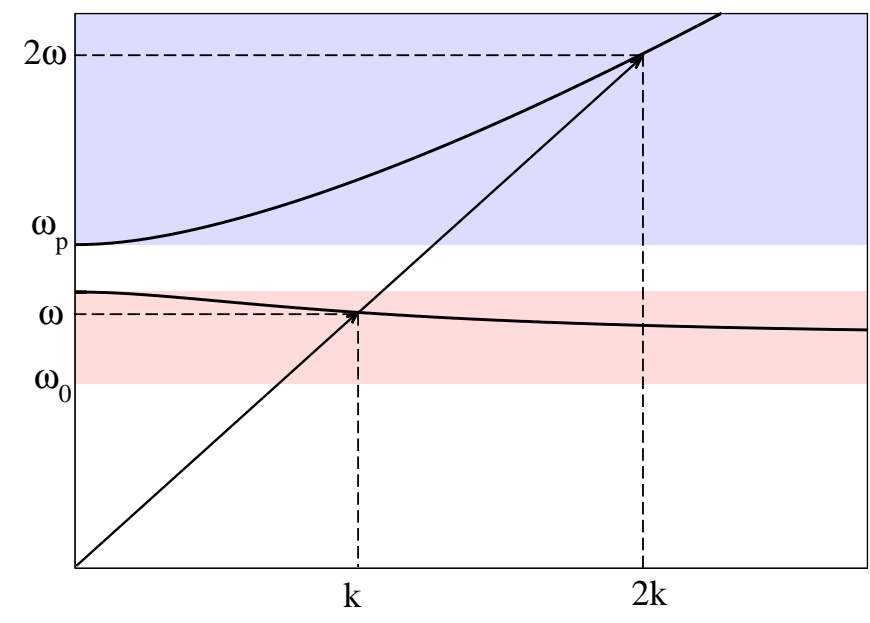

FIG. 2: Dispersion of plane waves $k(\omega)$ in the metamaterial. Arrows show the parameters of the FF and $\mathrm{SH}$ waves corresponding to the exact spatio-temporal phase matching.

$\epsilon(\omega) \mu(\omega)<0$. As a result, the field with the frequency $\omega$ from this range incident on a semi-infinite left-handed medium will decay exponentially from the surface inside the metamaterial. Taking into account Eqs. (11) and (2), the depth $\delta$ of this skin-layer can be found as

$$
\delta=\left(k_{\|}^{2}-\epsilon \mu \frac{\omega^{2}}{c^{2}}\right)^{-1 / 2}<\frac{\lambda}{17},
$$

where $k_{\|}$is the tangential component of the wavevector of the incident wave, and $\lambda$ is a free space wavelength. For the SH wave generated in this layer, the metamaterial becomes transparent. In this case, a thin slab of a metamaterial may operate as a nonlinear left-handed lens that will provide an image of the source at the second harmonics, as suggested recently in Ref. 10]. This case has also been mentioned in the earlier paper on the second-harmonic generation [7].

Case II. The FF wave is left-handed, whereas the $\mathrm{SH}$ wave is right-handed. Such a process is possible when $\omega_{p}<2 \omega_{0}$ (see Fig. 11). What is truly remarkable here is the possibility of exact phase-matching of the SHG parametric process, in addition to the cases discussed earlier in Ref. [7]. The phase-matching conditions for this parametric process are depicted in the dispersion diagram of Fig. 2 for the propagating waves in the metamaterial where the dispersion of the plane waves is defined by the relation

$$
D(\omega, k)=\left[k^{2}-\epsilon(\omega) \mu(\omega) \frac{\omega^{2}}{c^{2}}\right]=0
$$

The exact phase matching takes place when $2 k(\omega)=$ $k(2 \omega)$. Different signs of the slopes of the curves at the frequencies $\omega$ and $2 \omega$ indicate that one of the waves is forward, while the other wave is backward.

To study the SHG process in metamaterials we consider a composite structure created by arrays of wires and SRRs. To generate a nonlinear quadratic response of the metamaterial, we assume that each SRR contains a diode, as depicted schematically in the inset of Fig. 1 The diode is described by the current-voltage dependence,

$$
I=\frac{U}{R_{d}}\left(1+\frac{U}{U_{c}}\right)
$$

where $U_{c}$ and $R_{d}$ are the parameters of the diode, and $U$ is the voltage on the diode. Equation (66) is valid provided $U \ll U_{c}$, and it represents two terms of the Taylor expansion series of the realistic (and more complex) current-voltage characteristics of the diode.

Following the standard procedure, we consider two components of the electromagnetic field at the fundamental frequency $\omega$ and its second harmonics $2 \omega$, assuming that all other components are not phase matched and therefore they give no substantial contribution into the nonlinear parametric interaction. Subsequently, we write the general coupled-mode equations describing the simultaneous propagation of two harmonics in the dispersive metamaterial as follows,

$$
\begin{aligned}
& \Delta \mathbf{H}_{1}+\epsilon(\omega) \mu(\omega) \frac{\omega^{2}}{c^{2}} \mathbf{H}_{1}=-\sigma_{1} \mathbf{H}_{1}^{*} \mathbf{H}_{2}, \\
& \Delta \mathbf{H}_{2}+4 \epsilon(2 \omega) \mu(2 \omega) \frac{\omega^{2}}{c^{2}} \mathbf{H}_{2}=-\sigma_{2} \mathbf{H}_{2},
\end{aligned}
$$

where the indices "1", "2" denote the FF and SH fields, respectively; $\Delta$ is a Laplacian, and other parameters are defined as follows

$$
\begin{array}{r}
\sigma_{1}=\kappa / 2 R(\omega), \quad \sigma_{2}=\kappa / R^{*}(\omega), \\
\kappa=\frac{6 \pi\left(\pi a^{2}\right)^{3}}{d^{3} c^{5}}\left[\frac{\omega_{0}^{4} \omega^{2}}{U_{c} R_{d} R(\omega) R(2 \omega)}\right],
\end{array}
$$

where $R(\omega)=\omega_{0}^{2} \omega^{2}+i \gamma \omega$, the asterisk stands for the complex conjugation, $a, d$ are the radius of the SRRs and the period of the metamaterial, respectively, and $\gamma$ is the damping coefficient of the SRR. For simplicity, we assume that both $\mathrm{FF}$ and $\mathrm{SH}$ waves are of the same polarization, and therefore they can be described by only one component of the magnetic field. In this case, Eqs. (7) become scalar. In the derivation of Eqs. (77) we take into account the Lorentz-Lorenz relation between the microscopic and macroscopic magnetic fields [11]. Also, it is assumed that the diode resistance $R_{d}$ is much larger than the impedance of the SRR slit, i.e. $R_{d} \gg 1 / \omega C$, so that the resonant properties of the composite are preserved.

\section{SEMI-INFINITE METAMATERIAL}

First, we consider a semi-infinite left-handed medium and the SHG process for the wave scattering at the surface. We assume that a TM-polarized FF wave is incident on a LH material from the vacuum, as shown schematically in Fig. 3 Inside the metamaterial, the wave at 


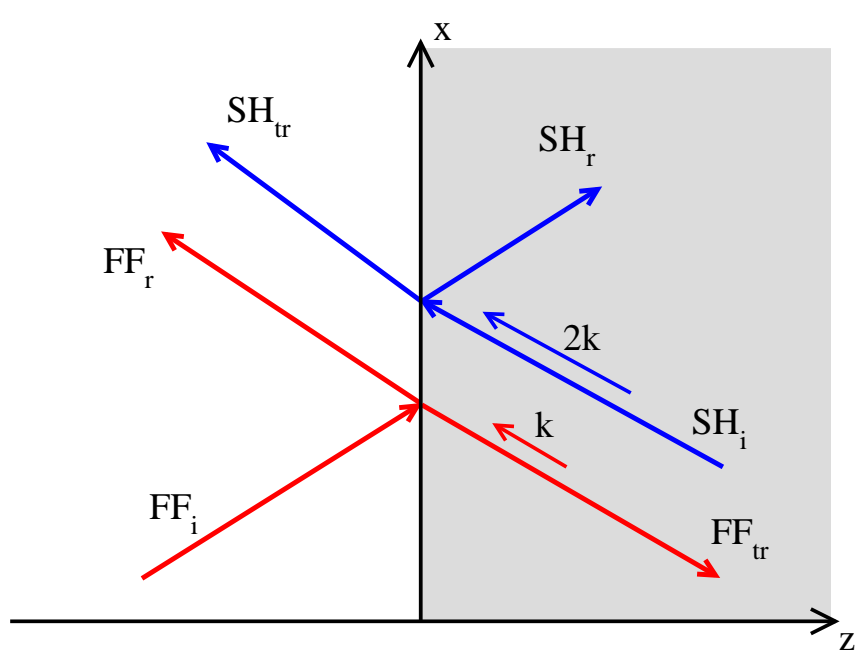

FIG. 3: Geometry of the SHG problem. Thick arrows show the direction of the energy flow, thin arrows - direction of wavevectors. Indices "i", "r", and "tr" stand for incident, reflected and transmitted waves, respectively.

the fundamental frequency satisfies the dispersion relation (5) which defines the wavenumber $k$. As is discussed above, the FF wave in the left-handed medium is backward, meaning that the normal component of the wave vector is directed towards the surface, i.e. in the direction opposite to the Poynting vector.

When the phase-matching conditions are satisfied, the generated $\mathrm{SH}$ wave has the wavevector parallel to that of the FF wave (see Fig. 3). However, the $\mathrm{SH}$ wave is forward propagating, so that the energy at this frequency should propagate towards the interface. When losses are negligible, the FF wave will be transformed completely into the $\mathrm{SH}$ wave with the energy flows in the direction opposite to that of the FF wave. This kind of the SHG process in a semi-infinite left-handed medium is characterized by two major features: (i) the efficiency of the SHG process may become very high, and (ii) the SH wave propagates in the direction opposite to that of the incoming FF wave.

\section{A. Coupled-mode equations}

To describe the SHG process analytically, we employ the coupled-mode theory and the slowly-varying envelope approximation for the FF and $\mathrm{SH}$ fields, and present the magnetic fields in the material in the form:

$$
H_{1,2}(t, z)=a_{1,2}(t, z) e^{i k_{1,2} z}+c . c .,
$$

where the amplitudes of the $\mathrm{FF}$ and $\mathrm{SH}$ fields $a_{1,2}(t, z)$ are assumed to vary slow in both space and time, i.e. $\partial a_{1,2} / \partial t \ll \omega a_{1,2}$, and $\partial a_{1,2} / \partial z \ll k a_{1,2}$. Substituting Eqs. (9) into Eqs. (7) and neglecting the second-order derivatives, we obtain the coupled equations,

$$
\frac{\partial a_{1}}{\partial t}+v_{g 1} \frac{\partial a_{1}}{\partial z}=i \sigma_{1} a_{1}^{*} a_{2}-\nu_{1} a_{1}
$$

$$
\frac{\partial a_{2}}{\partial t}+v_{g 2} \frac{\partial a_{2}}{\partial z}=i \sigma_{2} a_{1}^{2}+\nu_{2} a_{2}-i \Omega a_{2}
$$

where $v_{g 1,2}$ are the group velocities and $\nu_{1,2}=v_{g 1,2} \operatorname{Im}(k)$ are linear damping coefficients of the $\mathrm{FF}$ and $\mathrm{SH}$ fields, respectively,

$$
\Omega=q_{2} D(2 \omega, 2 k) / 2 \sigma_{2},
$$

is the phase mismatch, and

$$
q_{1}=\sigma_{1}\left[\frac{\partial D(\omega, k)}{\partial \omega}\right]^{-1}, \quad q_{2}=\sigma_{2}\left[\frac{\partial D(2 \omega, 2 k)}{\partial \omega}\right]^{-1}
$$

The coupled-mode equations (10) can be presented in the equivalent rescaled form,

$$
\begin{gathered}
\frac{\partial b_{1}}{\partial t}+v_{g 1} \frac{\partial b_{1}}{\partial z}=-q_{1} b_{1}^{*} b_{2}-\nu_{1} b_{1}, \\
\frac{\partial b_{2}}{\partial t}+v_{g 2} \frac{\partial b_{2}}{\partial z}=q_{2} b_{1}^{2}+\nu_{2} b_{2}-i \Omega b_{2}
\end{gathered}
$$

where $a_{1}=\alpha b_{1}, a_{2}=\beta b_{2}, \alpha=\exp (i \phi), \beta=$ $\exp [-i(\pi / 2-2 \phi)]$, and $\phi$ is an arbitrary phase.

The incoming FF backward travelling wave has the group velocity in the $z$-direction, and the phase velocity - in the opposite direction. The generated SH forward wave has both the phase and group velocities in the $-z$ direction. The FF wave propagates inside the material, and it loses the energy due to SHG and also due to losses in the medium. As a result, the $\mathrm{SH}$ amplitude decreases in the $z$-direction, and the boundary conditions should be taken in the form $b_{1,2}(\infty)=0$.

\section{B. Lossless process}

First, we neglect the metamaterial losses assuming, for simplicity, that $\nu_{1,2}=0$ and $\operatorname{Im}\left(q_{1,2}\right)=0$. At the large enough value of the phase mismatch we can neglect the derivatives in the second equation of the system (13), and obtain a local coupling between the FF and $\mathrm{SH}$ amplitudes,

$$
b_{2}=-i \frac{q_{2}}{\Omega} b_{1}^{2} .
$$

Substituting Eq. (14) into the equation for the FF field, we obtain a single nonlinear equation for the amplitude $b_{1}$ with an effective cubic nonlinearity,

$$
\frac{\partial b_{1}}{\partial t}+v_{g 1} \frac{\partial b_{1}}{\partial z}=i \frac{q_{1} q_{2}}{\Omega}\left|b_{1}\right|^{2} b_{1}
$$

The general solution of Eq. (15) can be found in the form

$$
b_{1}=A(t, z) e^{i \Phi(t, z)},
$$

where $A(t, z)=f\left(t-z / v_{g 1}\right), \Phi(t, z)=(t-z / a) q_{1} q_{2} f^{3}(t-$ $\left.z / v_{g 1}\right) / \Omega\left(1-v_{g 1} / a\right)$, where $f(t, z)$ is an arbitrary function, $a$ is constant, and $a \neq v_{g 1}$. The obtained solution describes a stationary nonlinear wave. 


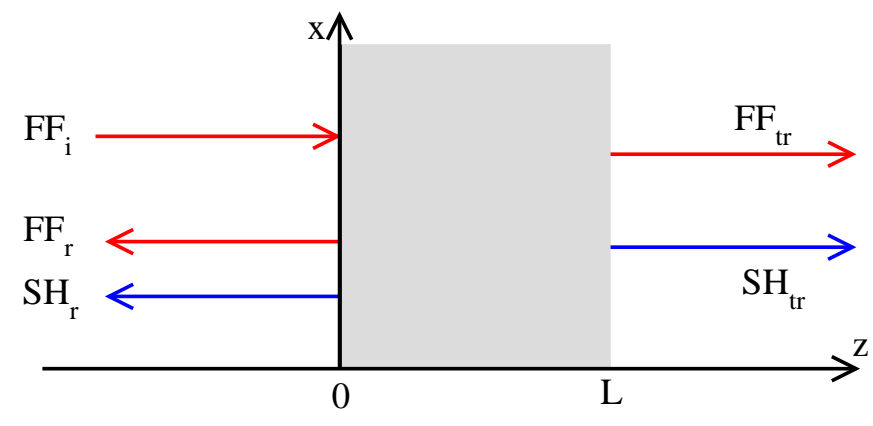

FIG. 4: Geometry of the SHG process for a finite-width slab of a nonlinear quadratic metamaterial (shaded).

In the case of the exact phase matching, i.e. when $\Omega=0$, Eqs. (13) are simplified,

$$
\begin{gathered}
\frac{\partial b_{1}}{\partial t}+v_{g 1} \frac{\partial b_{1}}{\partial z}=-q_{1} b_{1}^{*} b_{2} \\
\frac{\partial b_{2}}{\partial t}-v_{g 2} \frac{\partial b_{2}}{\partial z}=q_{2} b_{1}^{2}
\end{gathered}
$$

Looking for stationary solutions of Eqs. (17), we take $\partial / \partial t=0$ and find the integral of motion of this system,

$$
\frac{v_{g 1}}{q_{1}}\left|b_{1}\right|^{2}-\frac{v_{g 2}}{q_{2}}\left|b_{2}\right|^{2}=C
$$

Since both the waves should vanish inside the metamaterial, we take $C=0$. Then, the solution of Eqs. (17) is found as

$$
b_{1}=\left(\frac{v_{g 1} v_{g 2}}{q_{1} q_{2}}\right)^{1 / 2} \frac{1}{\left(z+z_{0}\right)}, \quad b_{2}=\frac{v_{g 1}}{q_{1}} \frac{1}{\left(z+z_{0}\right)},
$$

where $z_{0}$ is constant. Solution (19) has a singularity, and it corresponds to an explosive instability of the parametrically interacting waves. However, this singularity does not appear in the corresponding boundary problem.

Equations (17) describe also the solution for the coupled FF and SH fields, where the FF wave has a localized profile, while the $\mathrm{SH}$ wave has a tanh profile,

$$
\begin{aligned}
& b_{1}(t, z)=A_{1} \operatorname{sech}[(t-z / \beta) / T], \\
& b_{2}(t, z)=A_{2} \tanh [(t-z / \beta) / T],
\end{aligned}
$$

where

$$
A_{1}=\left[\frac{\left(v_{g 2}+\beta\right)\left(v_{g 1}-\beta\right)}{\beta^{2} T^{2} q_{1} q_{2}}\right]^{1 / 2}, A_{2}=\frac{\left(v_{g 1}-\beta\right)}{\beta T q_{1}}
$$

$\beta$ is the pulse velocity, and $T$ is a characteristic time scale. Solutions (20) are valid provided $\beta<-v_{g 2}$, which means that in the moving reference frame both waves should be of the same type: either forward or backward. The analytical solution (20) and (21) is characterized by two arbitrary constants, $\beta$ and $T$, so that the corresponding family of solutions is two-parametric.

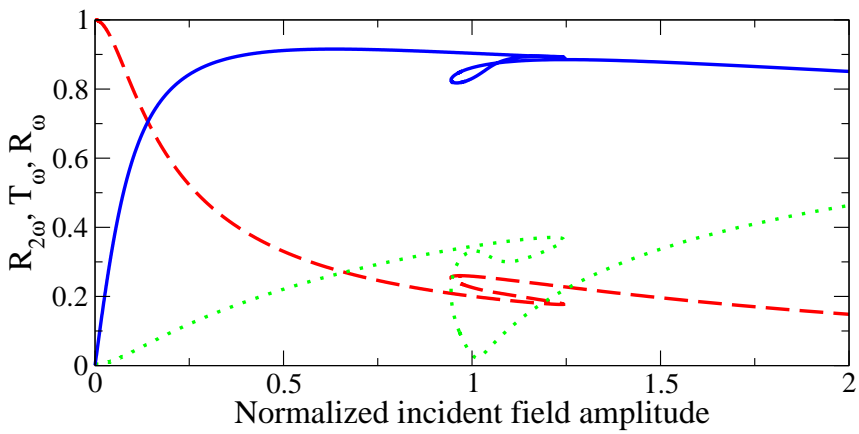

FIG. 5: Reflection coefficients of the generated SH wave (solid) and transmitted FF (dotted), as well as the transmission coefficient of the FF wave (dashed) vs. the normalized field intensity. Normalized slab thickness $L=10$.

\section{Dissipative process}

Next, we consider more realistic case with a linear damping of the $\mathrm{SH}$ wave smaller than that of the $\mathrm{FF}$ wave, since the FF wave is much closer to the resonance where such losses may become quite essential. We assume again $\operatorname{Im}\left(q_{1,2}\right)=0$, so that the stationary solution of Eqs. (13) can be found in this case analytically under the conditions of the exact phase matching. First, we find the integral of motion of the coupled system in the form,

$$
\frac{v_{g 2}}{v_{g 1}}\left[q_{1} b_{2}^{2}+2 \nu_{1} b_{2}\right]=q_{2} b_{1}^{2}
$$

Using this invariant, we find the solutions for the amplitudes of FF and $\mathrm{SH}$ waves as follows

$$
\begin{array}{r}
b_{1}(z)=\nu_{1}\left(\frac{v_{g 2}}{q_{1} q_{2} v_{g 1}}\right)^{1 / 2} \operatorname{csch}\left[\frac{\nu_{1}}{v_{g 1}}\left(z+z_{0}\right)\right], \\
b_{2}(z)=\frac{2 \nu_{1}}{q_{1}}\left[e^{2 \nu_{1}\left(z+z_{0}\right) / v_{g 1}}-1\right]^{-1} .
\end{array}
$$

In the lossless case, Eqs. 23 coincide with Eqs. (19), however, the presence of finite losses changes the type of solutions from power to exponential ones.

\section{A SLAB OF METAMATERIAL}

Next, we study the SHG process for a layer of the thickness $L$ (see Fig. 4) and employ a direct numerical approach to solve Eqs. (7). First, we rewrite Eqs. (7) in the dimensionless form

$$
\begin{gathered}
\frac{d^{2} H_{1}}{d z^{2}}+\left[\epsilon(\omega) \mu(\omega)-k_{x}^{2}\right] H_{1}=-H_{2} H_{1}^{*}, \\
\frac{d^{2} H_{2}}{d z^{2}}+4\left[\epsilon(2 \omega) \mu(2 \omega)-k_{x}^{2}\right] H_{2}=-Q H_{1}^{2},
\end{gathered}
$$

where the magnetic field is normalized by the value $\omega^{2} \sigma_{1} / c^{2}\left|\sigma_{1}\right|^{2}, z$ is normalized by the value $c / \omega$, and 


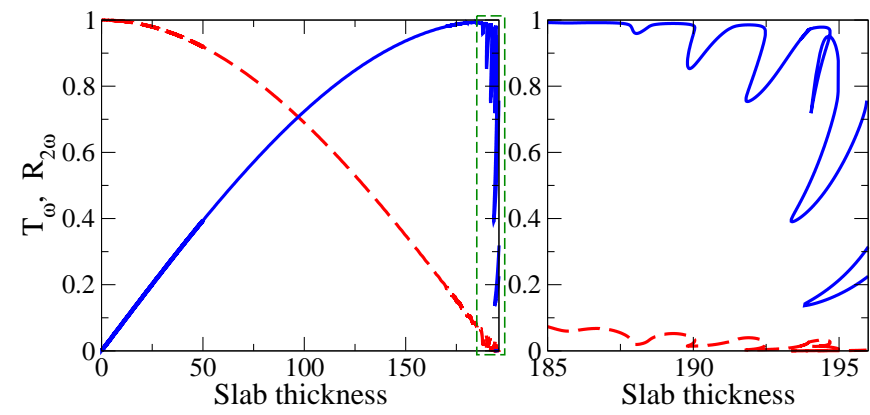

FIG. 6: Transmission coefficient of the FF wave (dashed) and reflection coefficient of the $\mathrm{SH}$ wave (solid) vs. the slab thickness $L$, for a fixed amplitude of the transmitted wave, $H_{1}(L)=10^{-2}$. Right plot shows a blow up of the region depicted by a dashed box.

$Q=\sigma_{1} \sigma_{2} /\left|\sigma_{1}\right|^{2}$. We assume that a slab of the lefthanded material is illuminated by the $\mathrm{FF}$ wave with the amplitude $H_{\omega}^{(i)}$, and the SH wave is generated inside the slab, so that the reflected and transmitted waves of both the frequencies $\omega$ and $2 \omega$ appear (see Fig. 4), with the amplitudes $H_{1}^{(t)}, H_{2}^{(t)}, H_{1}^{(r)}, H_{2}^{(r)}$, respectively. Solving the coupled-mode equations numerically, we present our results for the reflection and transmission coefficients defined as $R_{\omega, 2 \omega}=H_{1,2}^{(r)} / H_{1}^{(i)}$ and $T_{\omega, 2 \omega}=H_{1,2}^{(t)} / H_{1}^{(i)}$.

In the calculations presented here we assume that the left-handed material is lossless, and we consider the normal incidence. We take the following parameters of the composite: $\omega_{0}=2 \pi \times 5 \mathrm{GHz}, \omega_{p}=2 \pi \times 7 \mathrm{~s}^{-1}, F=0.3$, $a=3 \mathrm{~mm}, d=6 \mathrm{~mm}, U_{c} R_{d}=10^{5}$ CGS. For such parameters the exact phase matching takes place at $f_{p m} \approx 5.37$ GHz. Dependences of the coefficients $R_{2 \omega}, T_{\omega}$ and $R_{\omega}$ vs. the amplitude of the incident FF wave are shown in

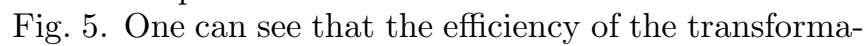
tion of the incident $\mathrm{FF}$ wave into the reflected $\mathrm{SH}$ wave can be rather high. Larger intensities of the incident field result in the multistable behavior of the reflection and transmission coefficients.

Figure 6] shows the dependence of the transmission coefficient of the FF wave and the reflection coefficient of the SH wave vs. the slab thickness, for a fixed amplitude of the transmitted wave. We observe multistable behavior of the coefficients for thicker slabs.

\section{CONCLUSIONS}

We have presented a comprehensive study of the specific features of the second-harmonic generation in lefthanded metamaterials with a quadratic nonlinear response. First, we have demonstrated a possibility of the exact phase matching between backward propagating FF and forward propagating SH waves. Then, we have developed an analytical approach based on a novel type of the coupled-mode equations in order to characterize the process of the harmonic generation from a semi-infinite left-handed metamaterial. In particular, we have demonstrated that a surface of the left-handed metamaterial can operate as an effective mirror that reflects all generated SH waves. Finally, we have performed numerical simulations of the second-harmonic generation process for a finite slab of the metamaterial, and revealed quite unusual multi-valued multistable transmissions and reflections.

\section{ACKNOWLEDGMENTS}

The work was supported by the Australian Research Council. Alexander Zharov acknowledges a warm hospitality of the Nonlinear Physics Centre during his stay in Canberra, as well as a support from the Russian Fund for Basic Research (grant N05-02-16357).
[1] V. G. Veselago, Sov. Phys. Solid State 8, 2854 (1967); V. G. Veselago, Usp. Fiz. Nauk 92, 517 (1967).

[2] D. R. Smith, W. J. Padilla, D. C. Vier, S. C. Nemat Nasser, and S. Schultz, Phys. Rev. Lett. 84, 4184 (2000); D.R. Smith, J.B. Pendry, and M.C.K. Wiltshire, Science 305, 788 (2004).

[3] R. A. Shelby, D. R. Smith, and S. Schultz, Science 292, 77 (2001).

[4] A. A. Zharov, I. V. Shadrivov, and Yu. S. Kivshar, Phys. Rev. Lett. 91, 037401 (2003).

[5] N. A. Zharova, I. V. Shadrivov, A. A. Zharov, and Yu. S. Kivshar, Optics Express 13, 1291 (2005).

[6] M. Lapine, M. Gorkunov, and K. H. Ringhofer, Phys.
Rev. E 67, 065601 (2003).

[7] V. M. Agranovich, Y. R. Shen, R. H. Baughman, and A. A. Zakhidov, Phys. Rev. B 69, 165112 (2004).

[8] M. Lapine and M. Gorkunov, Phys. Rev. E 70, 66601 (2004).

[9] A. A. Zharov, Fiz. Plazmy 17, 20 (1991).

[10] A. A. Zharov, N. A. Zharova, I. V. Shadrivov, and Yu. S. Kivshar, Appl. Phys. Lett. (2005) in press; a preprint is available at arXiv:physics/0404074 (2004).

[11] L. D. Landau and E. M. Lifshitz, Electrodynamics of Continuous Media (Pergamon Press, Oxford, 1963). 